\title{
Comparison of Antiobesity Effects of Adipose-Derived Stromal/Stem Cells from Different Sources in a Natural Aging Model
}

\author{
Yu Zhu ${ }^{1-3, *}$ \\ Tao Wang ${ }^{1-3, *}$ \\ Shuangli $\mathrm{He}^{1-3}$ \\ Shiming $\mathrm{Pu}^{\mathrm{I}-3}$ \\ Hongxia Zhao ${ }^{1-3}$ \\ Zuping Zhou ${ }^{1-3}$ \\ Qiong $\mathrm{Wu}^{\mathrm{I}-3}$
}

'School of Life Sciences, Guangxi Normal University, Guilin, Guangxi Zhuang Autonomous Region, People's Republic of China; ${ }^{2}$ Guangxi Universities Key Laboratory of Stem Cell and Biopharmaceutical Technology, Guangxi Normal University, Guilin, Guangxi Zhuang Autonomous Region, People's Republic of China; ${ }^{3}$ Research Center for Biomedical Sciences, Guangxi Normal University, Guilin, Guangxi Zhuang Autonomous Region, People's Republic of China

*These authors contributed equally to this work
Correspondence: Qiong Wu Tel +86 I8978327544

Email wubinbin02@I63.com
Purpose: Our previous study found that white adipose stem cells (W-ASCs) derived from abdominal and femoral sulcus white adipose stem cells (ASCs) have antiaging and agerelated obesity effects. Whether interscapular brown adipose stem cells (B-ASCs) have the same effect has not been reported. The study objective was to compare the effects of ASCs from different tissues on aging and aging-related obesity.

Patients and Methods: C57BL/6J mice at 22 months of age were transplanted with either B-ASCs or W-ASCs from young mice at 2 months of age. Changes in body weight, biochemistry, cytokines, hormone secretion, cell senescence, lipid metabolism, and ASC function were assessed after transplanted 1 month.

Results: W-ASCs were superior to B-ASCs as aging and age-related obesity indicators, based on change in body weight, organ weight, antioxidant and anti-inflammatory activity, lipid metabolism, and liver and kidney function.

Conclusion: Difference in the tissue source was reflected by the heterogeneity of antiaging and age-related obesity effects of transplanted ASCs. Based on the study results, we recommend W-ASCs over B-ASCs in aging and age-related obesity applications.

Keywords: adipose-derived stromal/stem cells, different tissue sources, obesity, aging

\section{Introduction}

Obesity is a problem from birth throughout aging. Increased visceral fat is one of the changes that accompanies aging, and changes in the abundance and distribution of adipose tissue, and in endocrine signals with aging can have negative outcomes, such as chronic inflammation and chronic diseases including type 2 diabetes. ${ }^{1,2}$ Regardless of such developments, research on how aging and obesity interact is incomplete.

The two forms of mammalian fat are white adipose tissue (WAT) and brown adipose tissue (BAT). ${ }^{3}$ WAT stores excess energy as triglycerides (TGs) to maintain energy metabolism. ${ }^{4}$ BAT consumes energy and releases it as heat. BAT cells are small and contain many mitochondria; and, in experimental animal studies, BAT has been found to inhibit the development of obesity. ${ }^{3-5}$ Adipose stem cells (ASCs) are easily obtained from adult adipose tissue, have high proliferative activity, and ability to differentiate is second only to embryonic stem cells. ${ }^{6}$ The primary role of ASCs is to maintain adipocyte homeostasis by self-renewal and differentiation. In addition, it is also related to immunity and metabolism. ${ }^{7,8}$

The correlation between aging and obesity is obvious, and ASCs are closely related to obesity. To investigate the role of ASCs in aging and obesity, we followed the 
changes in adipose stem cells isolated from WAT and BAT that occurred in a mouse model of aging The results indicated that the content of WAT was increased in old mice and the content of BAT was increased in young mice. The numbers of senescent cells and the rate of apoptosis of ASCs were significantly higher in old compared with young mice, and osteogenic and adipogenic differentiation capacity were decreased. ${ }^{9}$

Previous studies have reported the osteogenic differentiation of ASCs in young mice ${ }^{9,10}$ and that the intravenous injection of fat-derived stem cells with autologous ASCs effectively regulated the blood and biochemical parameters, renal function, and antioxidant enzyme activity of elderly rats. ${ }^{10}$ In this study, intraperitoneal injection of white adipose stem cells (W-ASCs) in young mice reduced the gain in body weight observed in old mice, increased the levels of antioxidant enzymes such as superoxide dismutase (SOD) and catalase (CAT), and decreased the levels of inflammatory factors such as tumor necrosis factor (TNF- $\alpha$ ) and interleukin-6 (IL-6). ${ }^{11}$ The study objectives were to determine whether, as an energyconsuming and heat-producing tissue, brown fat stem cells (B-ASCs) could antagonize age-related obesity and improve age-related obesity, and what the differences exist between them and W-ASCs.

\section{Materials and Methods}

\section{Animal Preparation and Transplantation}

C57BL/6J mice were obtained from the Department of Experimental Animal Stem Cells of Guangxi Normal University (Guilin, China). The study procedures approved by, and followed the Guidelines of, the Animal Care and Use Committee of Guangxi Normal University. The animals were raised under specific pathogen-free conditions and all were maintained with a 12-hour light/dark cycle at $23 \pm 2^{\circ} \mathrm{C}$, and free access to water and a standardized $10 \%$ lipid diet. The young and old mouse models were established as previously described. ${ }^{11}$ The young mice were 2 months of age; the old mice were 22 months of age. W-ASCs and B-ASCs were obtained from young mice. Thirty old mice were randomly assigned to three groups of ten each, with intraperitoneal injection of $10^{6} \mathrm{~W}$-ASCs or B-ASCs in the experimental groups and phosphate buffer solution (Sangon Biotech, E607008) in the control group, and the time of transplantation was 1 month.

\section{Isolation and Culture of W-ASCs and B-ASCs}

The isolation and culture of W-ASCs and B-ASCs have been previously described. ${ }^{9}$ The mice were anesthetized, and WAT was removed from the groin and BAT from the scapula with sterile surgical scissors and forceps after washing with phosphate buffered saline. After digestion, DMEM/F12 (Fisher Scientific, $8,115,296)$ medium containing $10 \%$ fetal bovine serum (Fisher Scientific, 1,715,752) was added, after the tissue was passed through a $70 \mu \mathrm{m}$ screen (BD Bioscience), the cells were centrifuged at $300 \mathrm{~g}$ for $5 \mathrm{~min}$ and the supernatant was discarded. The cells were washed twice with DMEM/F12 and centrifuged again at $300 \mathrm{~g}$ for $5 \mathrm{~min}$. The supernatant was discarded, and the resulting matrix and vascular components were resuspended in DMEM/F12 medium containing 10\% fetal bovine serum in cell-culture plates. The culture medium was changed after 36-48 hours, and the ASCs that had adhered to the plastic surface were cultured to $80 \%$ confluence. We examined the immunophenotyping (CD29 $/ \mathrm{CD} 44^{+} / \mathrm{CD} 105^{+} /$ $\mathrm{CD}^{-} 5^{-}$) of ASCs by flow cytometry, and Antibodies were from Thermo Fisher (Figure S1). The third generation of cells was used in the experimental procedures.

\section{Proliferation and Differentiation of ASCs}

The methods used to assay ASC proliferation and differentiation have been previously described. ${ }^{11}$ ASC proliferation was assayed with a methylthiazol tetrazolium (MTT) method and colony formation. ASC osteogenic and adipogenic differentiation were induced by culture in adipogenic and osteogenic media. Oil red (Solarbio, G1262) and alizarin (Solarbio, G8550 red were used to detect the formation of lipid droplets and calcium.

\section{Assay of Cellular Senescence}

ASCs from the transplantation and control groups were cultured for three generations and transferred at 10,000 cells/well to 96 -well plates. After 24 hours of culture, the cells that had adhered to the culture wells were fixed with paraformaldehyde and then stained following the manufacturer's (Sigma, CS0030MSDS) instructions. The senescent cells were counted cells and reported as a percentage of the total cell population.

\section{Serum Biochemical and Mitochondrial Assays}

Serum biochemistry and mitochondrial membrane potential were assayed as previously described. ${ }^{11}$ Whole blood was collected from the eyelid and centrifuged at 12,000 rpm at 
$4^{\circ} \mathrm{C}$ for $15 \mathrm{~min}$. The serum was collected and stored at $-80^{\circ} \mathrm{C}$. Serum TG, total cholesterol, high-density lipid (HDL) cholesterol, low-density lipid (LDL) cholesterol, glucose (GLU), blood urea nitrogen (BUN), glutamic acid transaminase (ALT), glutamic acid transaminase (AST) and creatinine (CREA) were determined with an autoanalyzer (HITACHI720). Serum SOD, catalase (CAT), sex hormone-binding globulin (SHBG), and thyroid stimulating hormone (TSH) were determined with enzyme-linked immunosorbent assay kits ( $\mathrm{R}$ \& D Systems). Mitochondrial membrane potential was measured with a mitochondrial dark red fluorescent probe following the manufacturer's instructions (Yeasen, 40743ES50).

\section{Tissue Weight and Histology}

After collecting blood, abdominal adipose tissue, liver, kidney, and spleen were removed and weighed. Liver tissue was fixed in neutral buffered formalin, processed, embedded in paraffin, sectioned, and stained with hematoxylin and eosin (Solarbio, G1120) or Oil Red O (Solarbio, G1262). Light microscopy and ImageJ version 5.0 software were used to measure the size of adipocytes and stored lipid droplets. ${ }^{11}$

\section{Cytokine Analysis}

A Luminex system (Millipore, Burlington, MA, USA) and mouse cytokine kits were used to assay tumor necrosis factor (TNF)- $\alpha$, interleukin (IL)-1 $\beta$, IL-6, chemokine ligand 2 (CCL2), C-X-C motif chemokine ligand 12 (CXCL12), epidermal growth factor (EGF), vascular endothelial growth factor (VEGF), fibroblast growth factor (FGF), and leptin (LEP). The samples were assayed in duplicate. $^{7}$

\section{Labeled ASCs}

ASCs were labeled with 4', 6-diamidine-2-phenyl indole (DAPI) and observed by blue fluorescence microscopy. Labeled cells were then transplanted into aging mice by intraperitoneal injection. Seven days later, abdominal fat was removed, embedded in optimal cutting temperature compound, and frozen sections were observed by light microscopy.

\section{Statistical Analysis}

Results were reported as means and standard deviation and $P$-values $<0.05$ was considered statistically significant. Data were analyzed using GraphPad Prism 9.0 for $t$ - tests or two-way analysis of variance.

\section{Results}

Changes in Body Weight, Visceral Fat Weight, Abdominal Fat Weight, and ASC Homing

Body fat, especially visceral fat, increases during aging. ${ }^{12,13}$ B-ASCs and W-ASCs from young mice were transplanted into naturally aging mice. The same volume of phosphate buffered saline was transplanted into the control group. The weight changes of that occurred in the aging mice within 4 weeks after injection are shown in (Figure 1A). The weight of mice transplanted with ASCs decreased in the first 2 weeks; the weight of mice in the control group increased. The weight of mice in the transplant groups increased in the last 2 weeks, but the rate of weight gain was lower than that of the control group. After 4 weeks, the mean weight in the W-ASC transplantation group $(25.2 \pm 0.6 \mathrm{~g})$ was $18.7 \%$ less than that of the control group $(31.0 \pm 1.0 \mathrm{~g})$. The mean weight in the B-ASC transplantation group $(27.4 \pm 0.4 \mathrm{~g})$ was $11.5 \%$ lower that of the control group. There were no differences in food intake between the control group and the transplantation groups (Figure 1B).

To explain the changes that occurred, we observed the DAPI-labeled ASCs 7 days after transplantation of into aging mice in frozen sections of abdominal fat (Figure 1C). We also weighed the internal organs and abdominal fat in each group (Figure $1 \mathrm{D}$ and $\mathrm{E}$ ). Compared with the control group $(0.26 \pm 0.24 \mathrm{~g})$, the weight of the spleen in the aging mice had decreased by $50.00 \%$ after B-ASC transplantation $(0.13 \pm 0.3 \mathrm{~g})$ and by $69.2 \%$ after W-ASC transplantation. Transplantation of B-ASCs had no significant effects on the weight of the kidneys or livers of naturally aging mice. The kidney weight decreased by $43.86 \%$ and the liver weight decreased by $33.91 \%(P<0.001)$ after W-ASC transplantation compared with the control group. Compared with the control group, the weight of the abdominal fat weight was reduced by $28.95 \%$ after transplantation of B-ASCs and by $52.63 \%$ after transplantation of W-ASCs $(P<0.01)$.

\section{Effects of B-ASCs and W-ASCs on Lipid Metabolism}

To investigate the effects of ASC transplantation on lipid metabolism during aging, we measured the size of the lipid droplets in abdominal fat, mitochondrial activity, and serum 
A

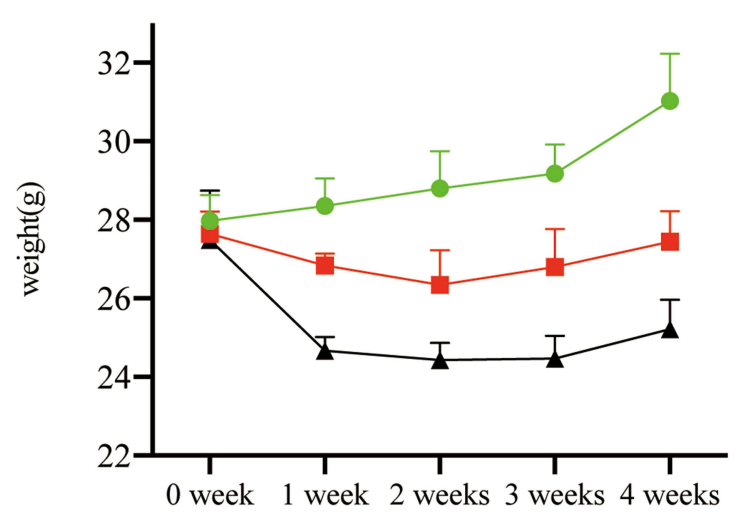

B

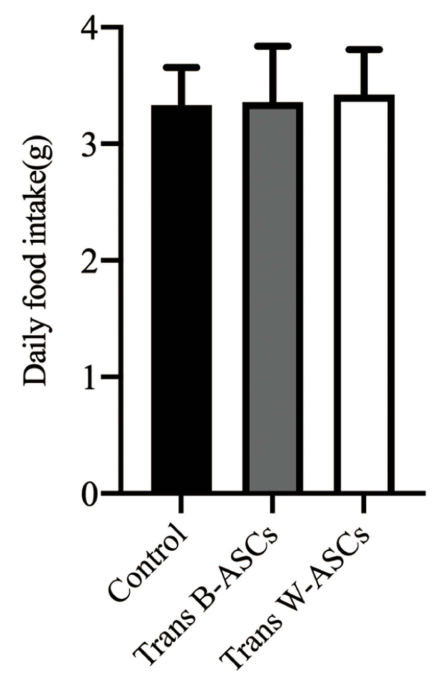

C

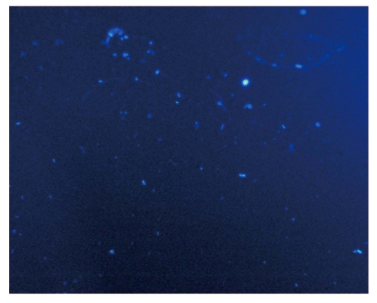

B-ASCs

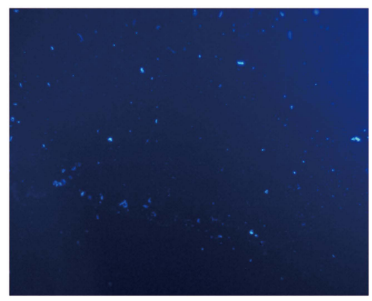

W-ASCs

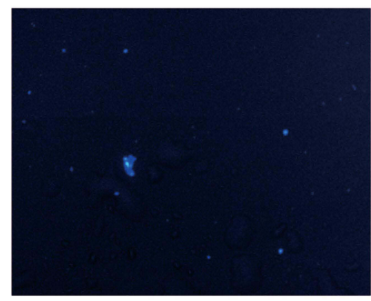

Trans B-ASCs

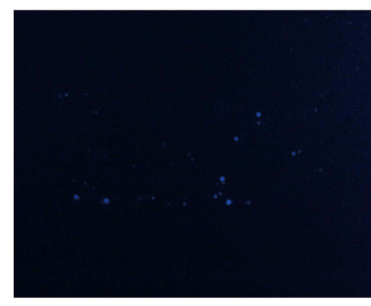

Trans W-ASCs
D

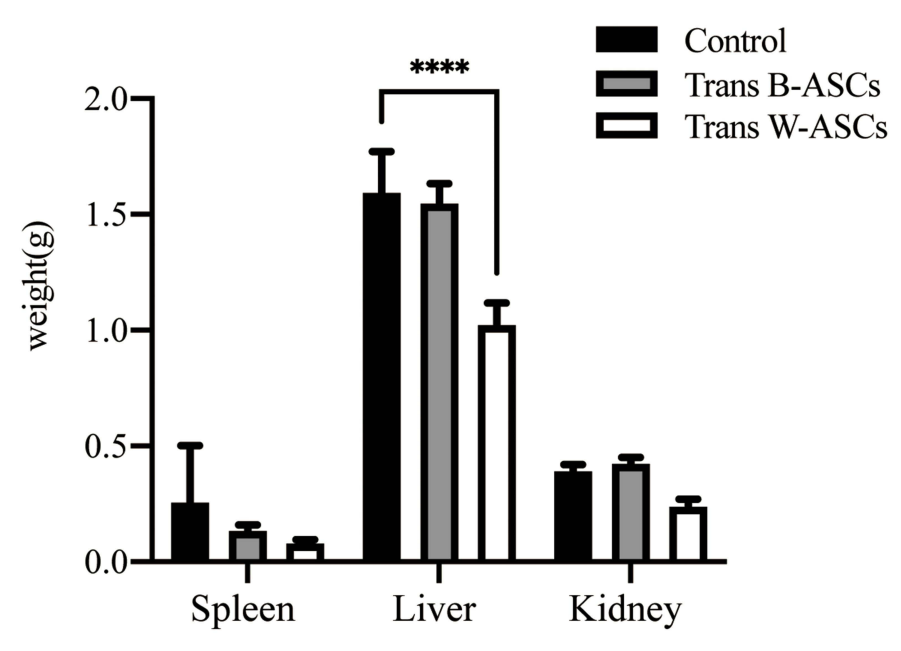

E

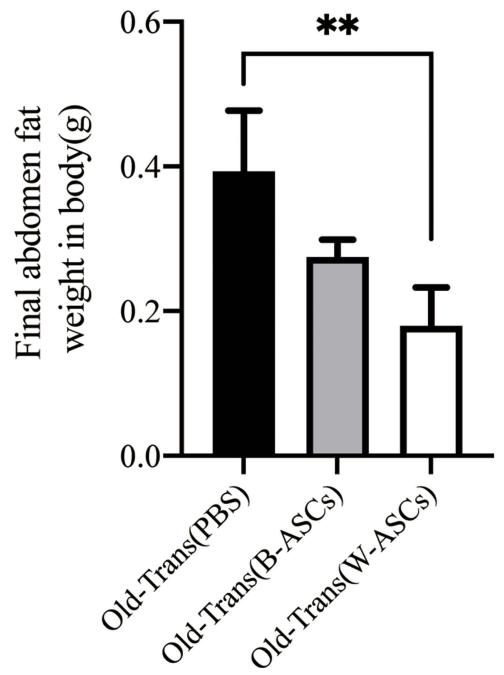

Figure I Weight-related changes and transplantation of labeled B-ASCs and W-ASCs. (A) Body weight. (B) Daily food intake. (C) Labeled ASCs (I00x). (D) Visceral fat weight. (E) Abdominal fat weight. Data are from four independent experiments $(n=10)$ and reported as means \pm standard deviation. Statistical analysis was conducted with GraphPad Prism 9 software.**p $<0.01$; ****p $<0.0001$. 
lipid levels. Hematoxylin and eosin staining of paraffinembedded sections of abdominal fat showed that the diameter of fat droplets in adipose cells was significantly smaller in aging mice after B-ASC and W-ASC transplantation than in the control group (Figure 2A). The fat droplet area was measured by Image $\mathrm{J}$ (Figure 2B); the differences significant $(P<0.05$ for B-ASCs and $P<0.001$ for $\mathrm{W}$-ASCs $)$. Both $\mathrm{B}-\mathrm{ASC}$ and $\mathrm{W}$-ASC transplantation significantly increased the TG level of aging mice $(P<0.05)$, and decreased GLU and HDL cholesterol (both $P<0.0001$ ). There was no significant effect on total cholesterol and LDL cholesterol (Figure 2C). There was thus no overall difference in the effects of B-ASC and W-ASC transplantation on the blood lipid levels of naturally aging mice. ASC transplantation enhanced mitochondrial activity, and the effect of B-ASC transplantation was stronger than that of W-ASCs (Figure 2D).

\section{Effects of B-ASC and W-ASC Transplantation on Aging Markers}

The effects of ASC transplantation were evaluated by agerelated oxidative stress, enzyme activity, and cellular senescence. Compared with the control group, SOD and CAT levels were increased by B-ASC and W-ASC transplantation. The differences in SOD level were significant for both types of ASCs $(P<0.001)$, but the difference in CAT levels was significant for only W-ASCs $(P<0.01)$. Sex hormone-binding globulin (SHBG) and TSH were both increased by ASC transplantation, but the difference was significant only for $\mathrm{W}-\mathrm{ASC}$ s $(P<0.01$ and $P<0.05$, respectively, Figure $3 \mathrm{~A})$. The $\beta$-galactosidase cell counts in the B-ASC $(P<0.01)$ and the W-ASC $(P<0.001)$ transplantation groups were significantly lower than the count in the control group (Figure 3B). In general, ASC transplantation reversed some of the aging criteria, and the W-ASCs had a greater effect than the B-ASCs.

\section{Effects of B-ASC and W-ASC Transplantation on Serum Cytokines}

Aging is usually accompanied by chronic systemic inflammation. ${ }^{14}$ The influence of ASCs on the extent of inflammation in naturally aging mice was evaluated by the levels of some serum cytokines (Figure 4). Compared with the control group, TNF- $\alpha$ was $48.41 \%$ lower in B-ASC transplantation group and $52.64 \%$ in the W-ASC group (P $<0.05)$. The corresponding differences in IL-6 level were $62.83 \%$ and $84.86 \%$, respectively, but neither were significant. LEP was significantly increased in the W-ASC group $(\mathrm{P}<0.001)$ and VEGF was significantly decreased $(P<0.05)$ compared with the control group. The differences in LEP and VEGF levels between the B-ASC group and the control group were not significant. Differences between IL-1 $\beta$, CCL12, CXCL12, EGF, and FGF in the control and ASC transplantation groups were not significant.

\section{Effects of B-ASCs and W-ASCs on the Plasticity of Abdominal ASCs in Naturally Aging Mice}

The relationships between abdominal fat loss and changes in abdominal-ASC function were investigated in ASCs isolated from abdominal fat. ASC proliferation was evaluated by an MTT assay and colony formation. The results (Figure 5A and $\mathrm{B}$ ) showed that there were no differences between the control and the transplantation groups. The effects on osteogenic and adipogenic differentiation are shown in Figure 5C and D. W-ASCs significantly enhanced osteogenic differentiation of abdominal ASCs $(\mathrm{P}<0.05)$; the effect of B-ASCs was not significant. Compared with the control group, both B-ASCs and W-ASCs significantly reduced adipogenic differentiation by abdominal ASCs in naturally aging mice $(\mathrm{P}<0.01$ and $\mathrm{P}<0.001$, respectively).

\section{Effects of B-ASCs and W-ASCs on Liver and Kidney Function of Naturally Aging Mice}

The effects of ASC transplantation on liver function were evaluated by ALT and AST levels (Figure 6A). Compared with the control group, ALT decreased in both transplantation groups, but the differences were not significant The reduction in AST was significant in both the B-ASC $(P<$ $0.001)$ and W-ASC groups $(P<0.01)$. The effects of ASC transplantation on renal function were measured by CREA and BUN levels (Figure 6B). Compared with the control group, CREA was significantly reduced in both the B-ASC $(P<0.05)$ and the W-ASC $(P<0.001)$ groups; the differences in BUN levels were not significant. Liver lipid accumulation was evaluated by histological staining (Figure 6C and D). Accumulation of lipid and lipid droplets was significantly reduced in both transplantation groups. The results indicate that ASC transplantation did not damage liver and kidney function, and improved liver and kidney function of aging mice to a certain extent. 
A

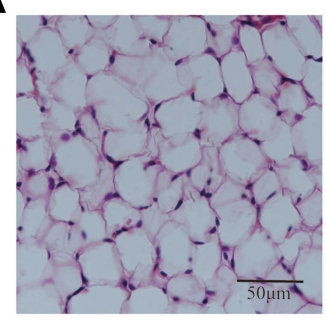

C

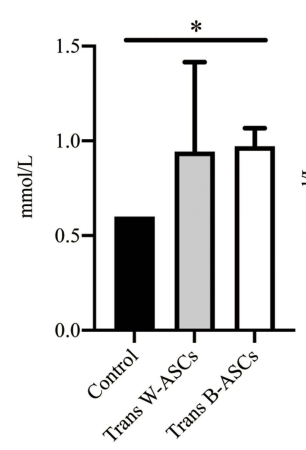

TG

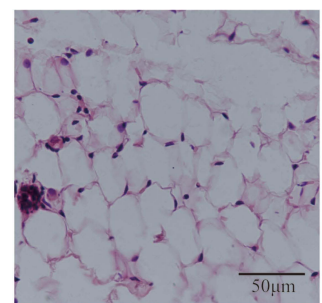

Old-Trans(B-ASCs)

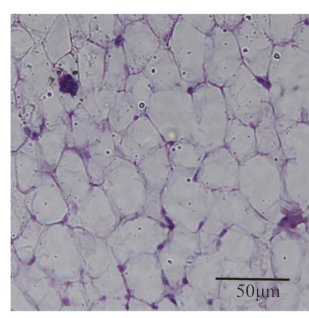

Old-Trans(W-ASCs)
B

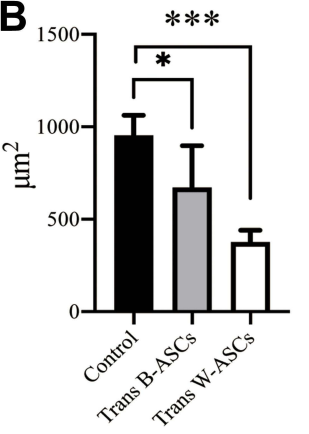

D

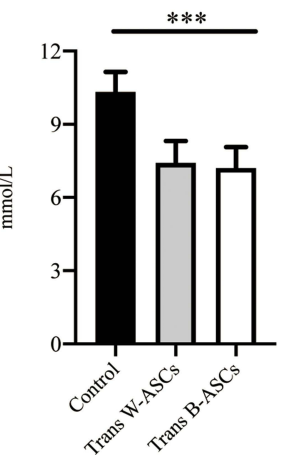

GLU

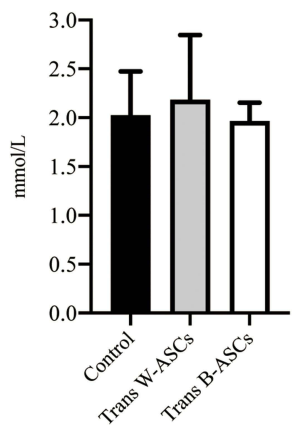

TCHO

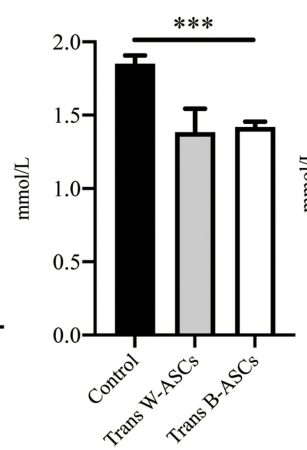

HDL

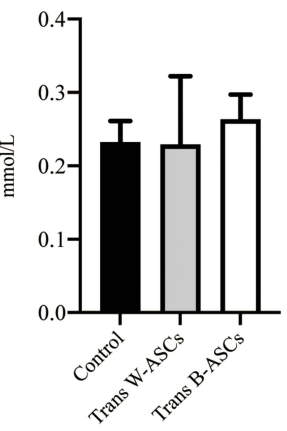

LDL
Control

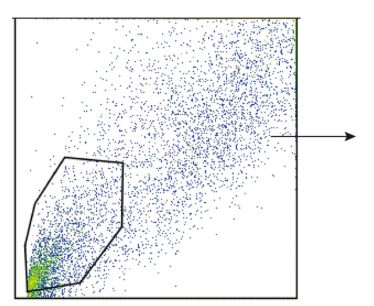

Trans B-ASCs

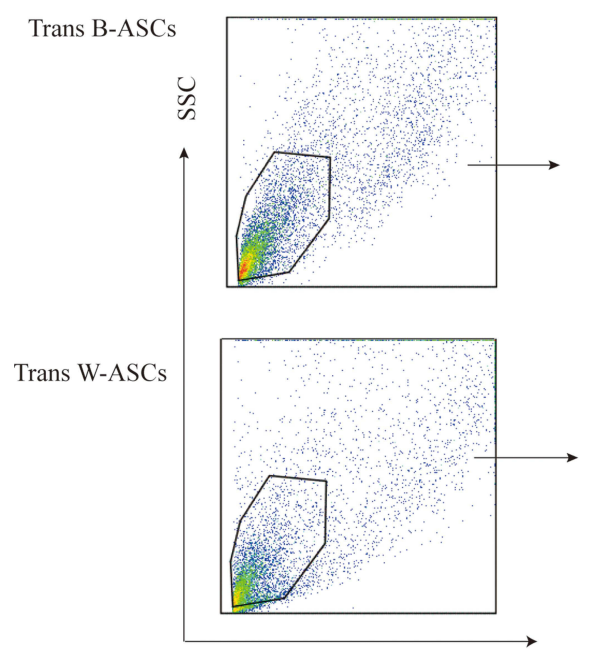

FSC
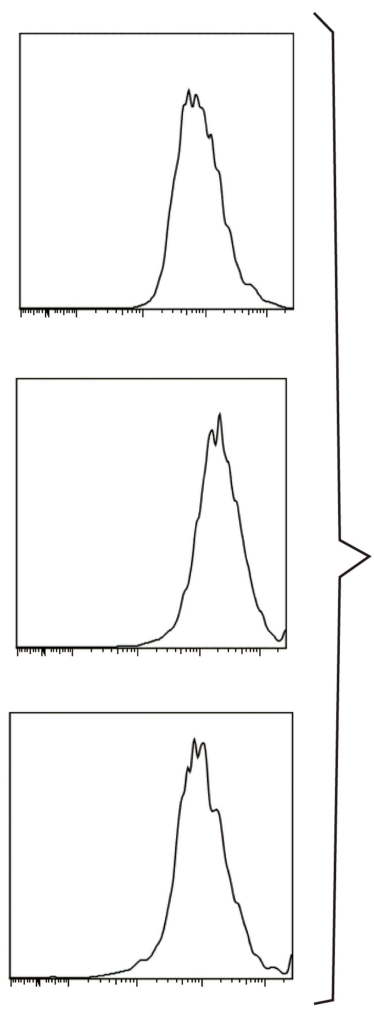

Control

Trans B-ASCs

_ Trans W-ASCs

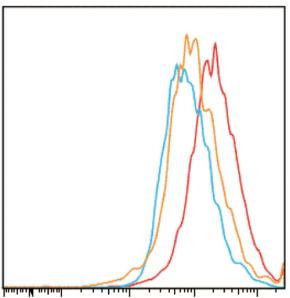

Figure 2 Effect of B-ASC and W-ASC transplantation on lipid metabolism. (A) Hematoxylin and eosin staining of paraffin-embedded abdominal adipose tissue. (B) The size of fat droplets in abdominal adipose tissue. (C) Blood lipid levels. (D) Mitochondrial activity in abdominal adipocytes. Data are from four independent experiments $(n=5)$ and reported as means \pm standard deviation. Statistical analysis was conducted with GraphPad Prism 9 software. $* P<0.05 ; * * * P<0.001$. 
A

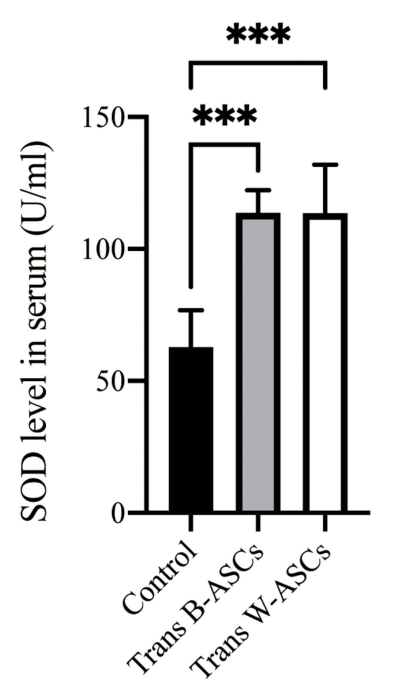

B

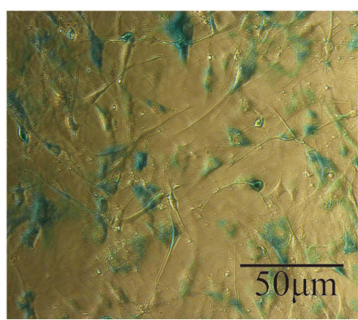

Control
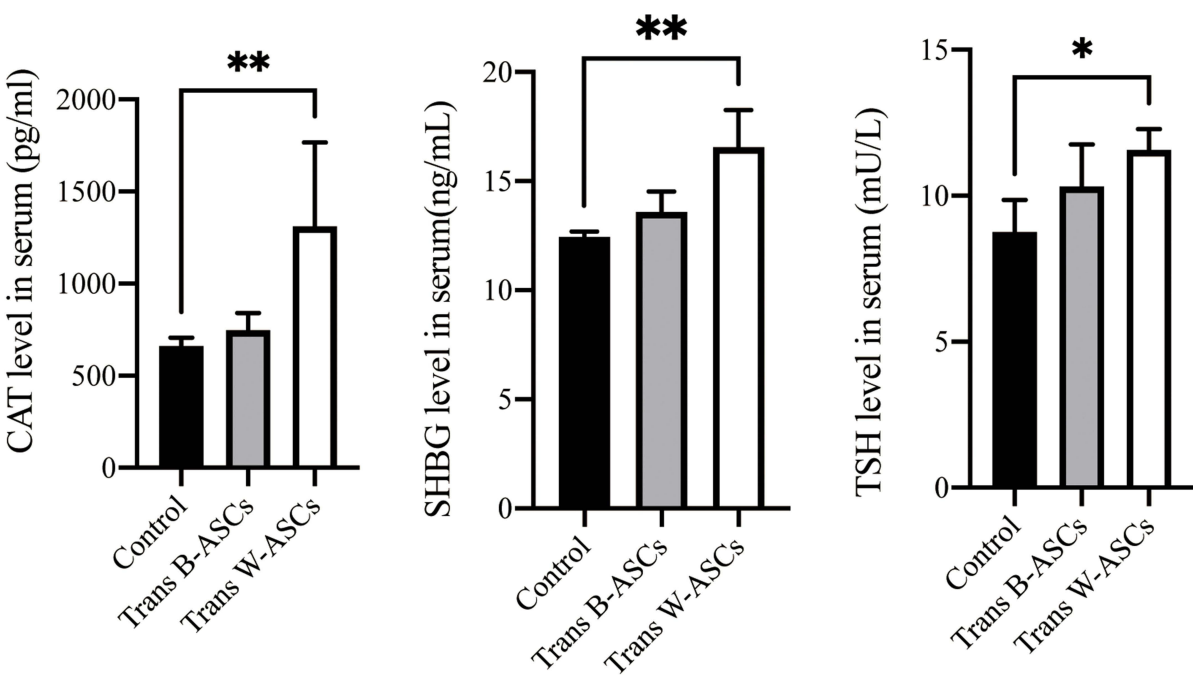

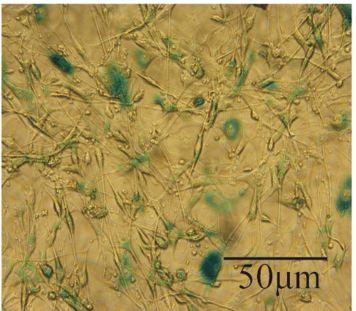

Trans B-ASCs

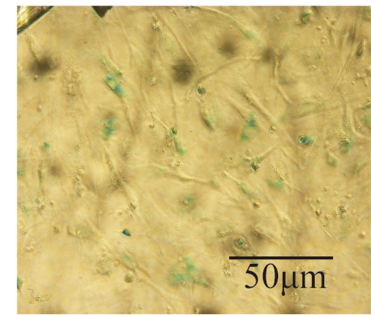

Trans W-ASCs

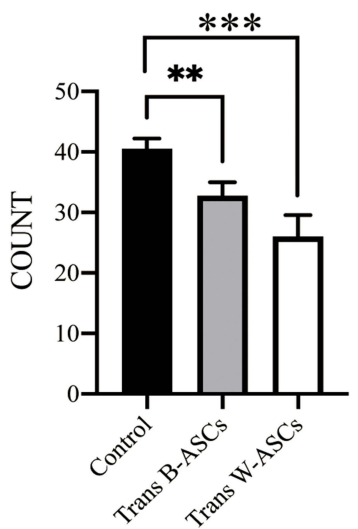

Figure 3 Antiaging effects of B-ASC and W-ASC transplantation. (A) Serum levels of superoxide dismutase (SOD), catalase (CAT), sex hormone-binding globulin (SHBG), and thyroid stimulating hormone (TSH). (B) Senescence-associated beta-galactosidase staining of ASCs from all study groups. Data are from four independent experiments $(n=7)$ and reported as means \pm standard deviation. Statistical analysis was conducted with GraphPad Prism 9 software. $* P<0.05 ; * * P<0.01$; $* * * P<0.00$ I.

\section{Discussion}

Our previous studies found that ASCs derived from inguinal white fat had the effect of resisting age-related obesity and improved age-related characteristics. ${ }^{11}$ The evidence supported the potential effectiveness of ASC transplantation for the treatment of aging and aging-related obesity. However, cells isolated from different sources and in different physiological states are likely to produce different therapeutic effects. ${ }^{15,16}$ Previous studies have shown that CRISPR-engineered human brown fat cells were able to prevent diet-induced obesity and improve metabolic syndrome in mice, ${ }^{17}$ but the effect of B-ASCs on aging and age-related obesity is not clear. Therefore, we compared the effects of inguinal W-ASCs from scapular B- ASCs from the scapula in the treatment of aging and age-related obesity. Both B-ASCs and W-ASCs had an effect on the reversal of age-related obesity and improvement of age- related characteristics, but the overall effects of B-ASCs were not as good as those of W-ASCs.

Both B-ASCs and W-ASCs reversed the increase in body weight of aging mice, which may be explained by decreases in abdominal fat content and abdominal organ weight. B-ASCs and W-ASCs reduced the abdominal fat weight of aging mice, and W-ASC but not B-ASC transplantation significantly reduced the spleen and liver weights. The difference might have been related to differences in the reduction of inflammation mediated by B-ASCs and W-ASCs. The weight of both the spleen and liver increase with age, ${ }^{18-20}$ and B-ASCs had less of an effect on age-related inflammation than that W-ASCs did. The serum cytokine results were consistent with the effects on inflammation, but the reasons need further investigation. We confirmed that the observed changes were not caused by differences in the amount of food intake; there were no differences in food intake among the 
TNF- $\alpha$

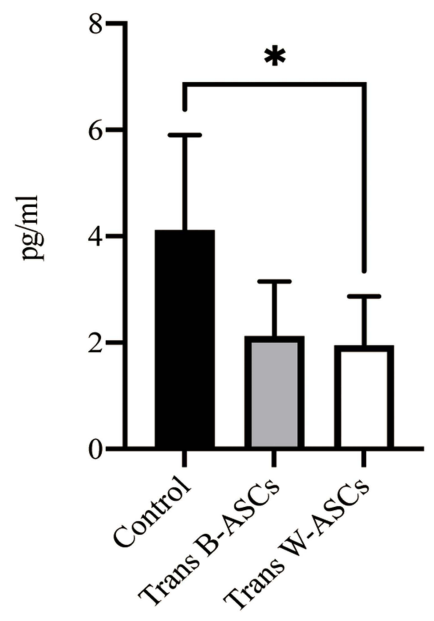

CCL2

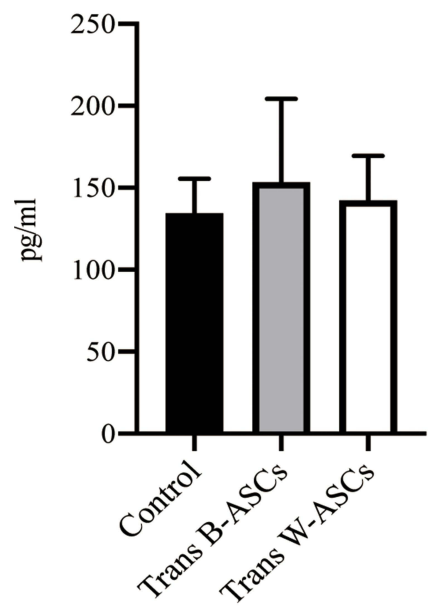

EGF

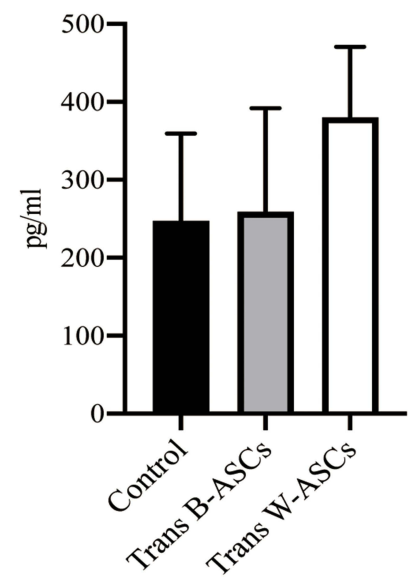

IL-1 $\beta$

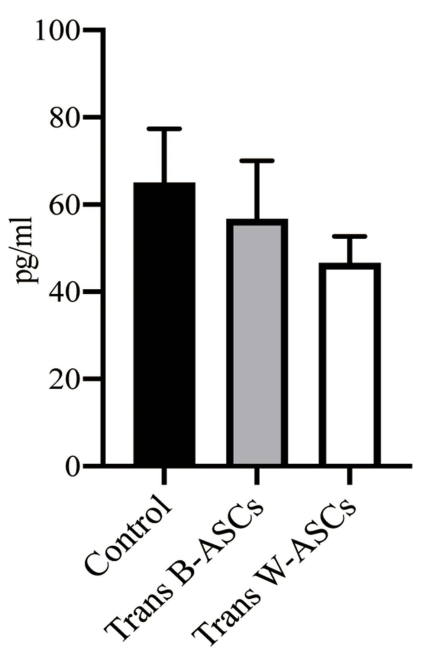

CXCL12

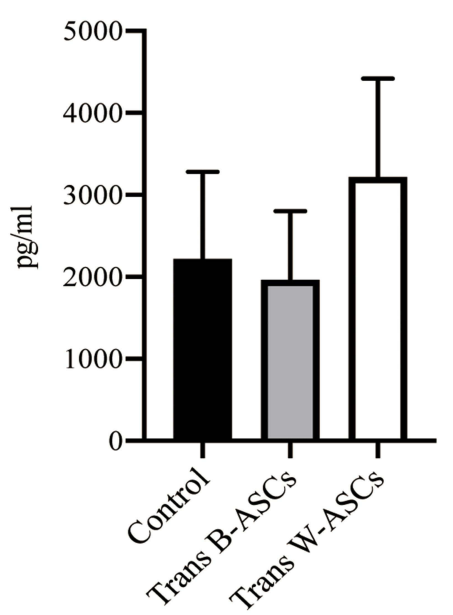

FGF

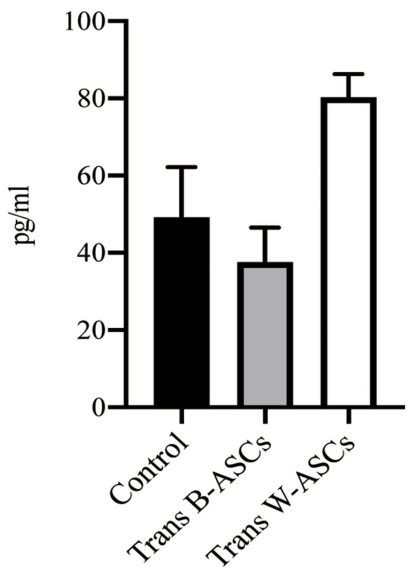

IL-6

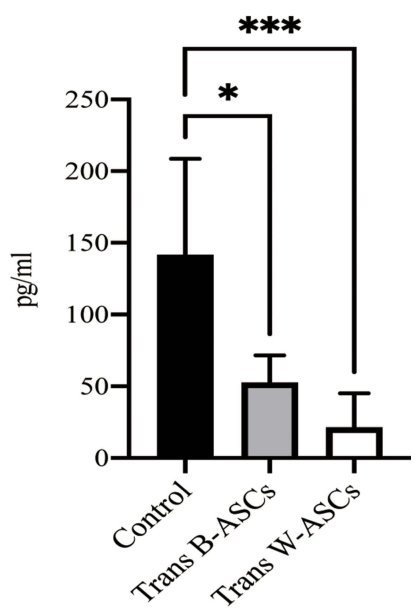

LEP
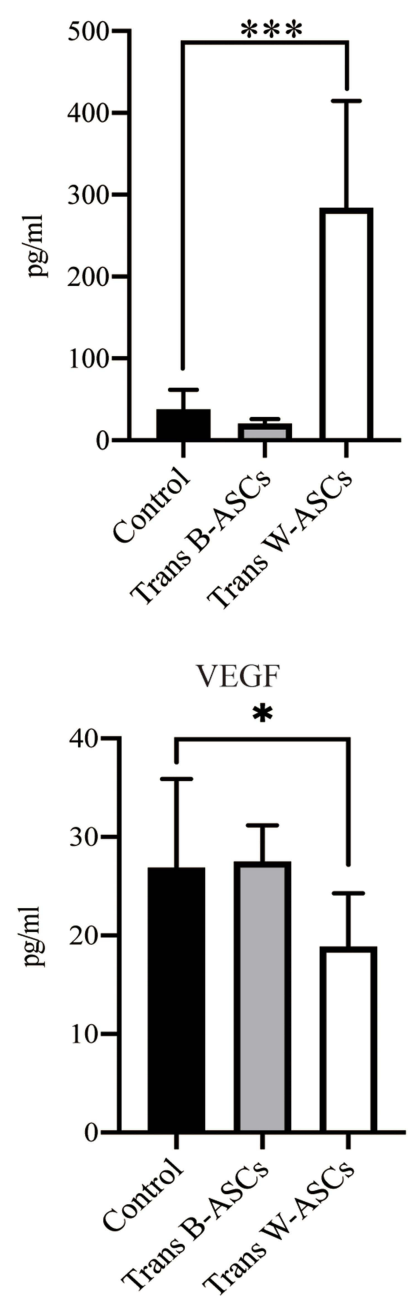

Figure 4 Serum cytokine concentrations. Data are mean \pm standard deviation $(n=8)$. Statistical analysis was conducted with GraphPad Prism $9 . * P<0.05 ; * * * P<0.001$. 
A
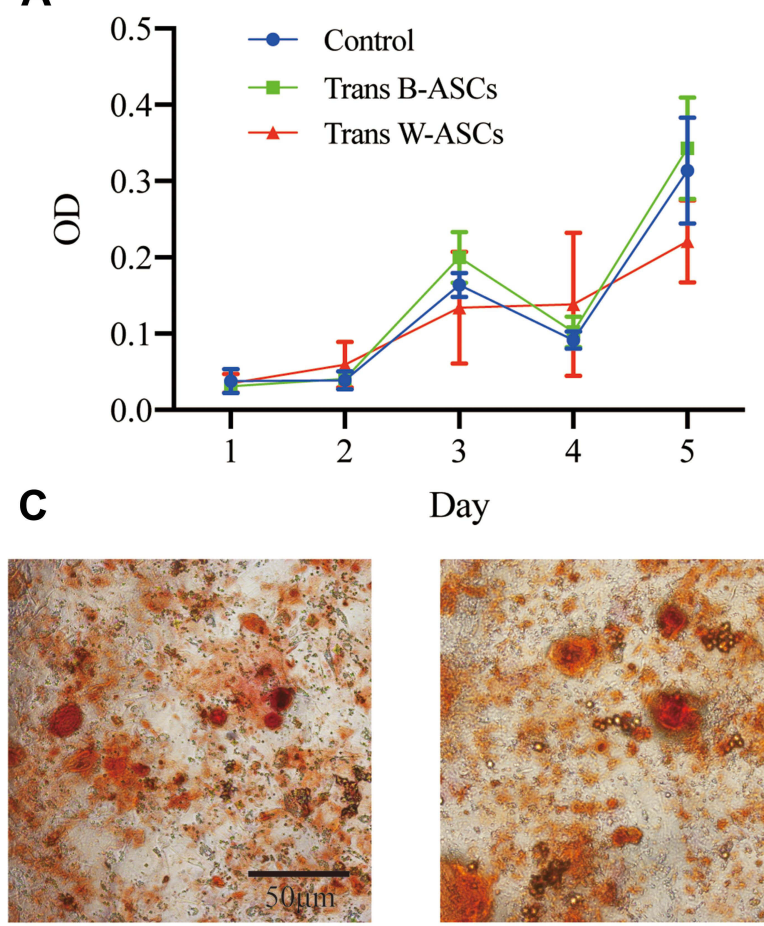

Control

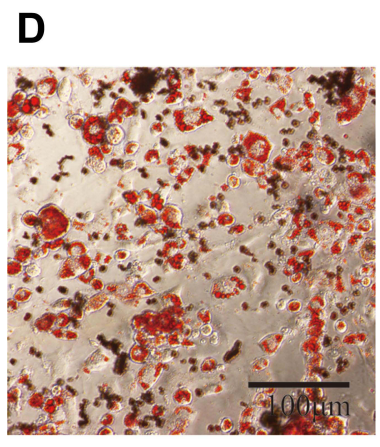

Control

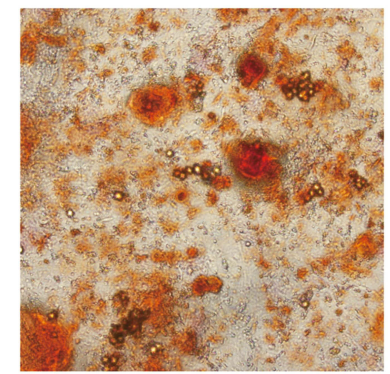

Trans B-ASCs

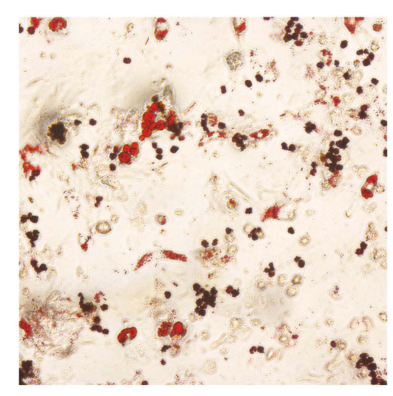

Trans B-ASCs
B

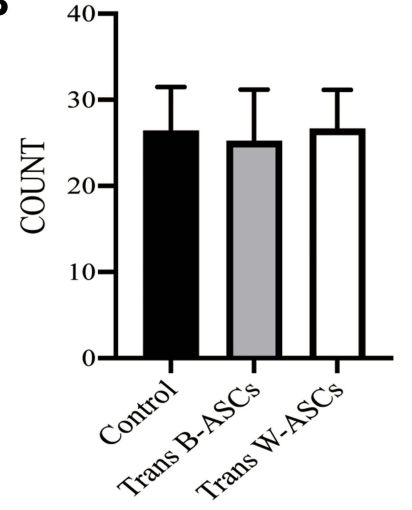

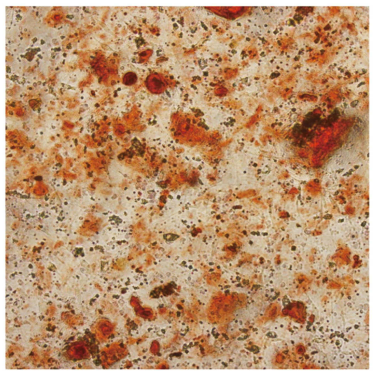

Trans W-ASCs

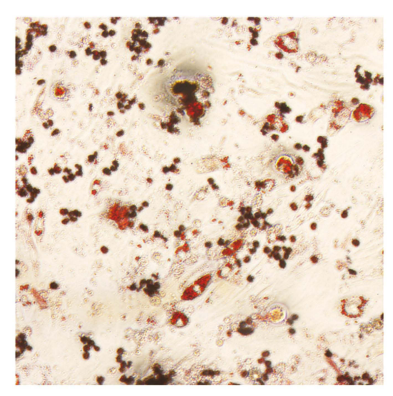

Trans W-ASCs
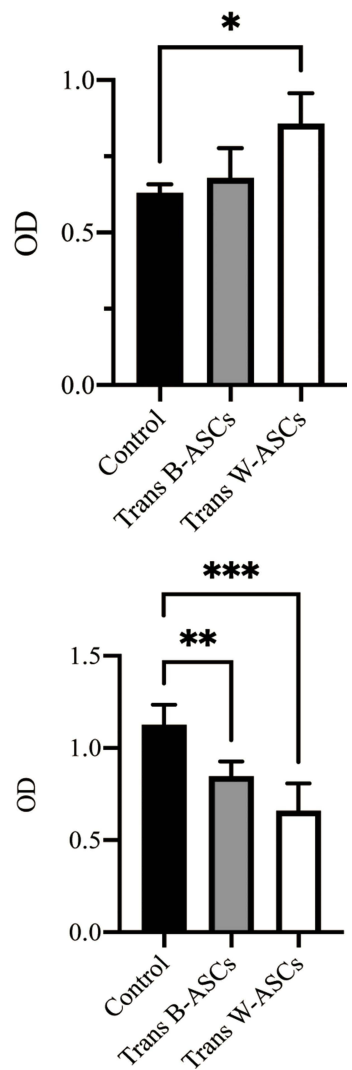

Figure 5 Effects of B-ASC and W-ASC transplantation on the plasticity of abdominal ASCs. (A) MTT assay of cell proliferation. (B) Colony count. (C) Osteogenic differentiation. (D) Adipogenic differentiation. Data are means \pm standard deviation $(n=5)$. Statistical analysis was conducted with $G$ raphPad Prism $9 . * P<0.05 ; * * P<0.0$ I; and $* * * P<0.001$.

groups. In addition, labeled B-ASCs and W-ASCs were found in abdominal fat tissue, which shows that the homing of transplanted ASCs to abdominal fat tissue played a role; reduction of abdominal fat weight and differentiation of abdominal ASCs also reflects this point.

Increased oxidative stress is an aging characteristic of aging, and oxidative damage accelerates the occurrence of some age-related diseases. High levels of oxidative stress can also accelerate the aging of mesenchymal stem cells and reduce their ability to differentiate into osteoblastic cells. ${ }^{21,22}$
B-ASC and W-ASC transplantation reduced oxidative stress in aging mice, which also explained the increase in the number of abdominal ASCs with aging and osteogenic differentiation capacity in the transplantation groups, but the improvement in oxidative stress in aging mice was less with B-ASCs than with W-ASCs. Decreases of SHBG and TSH also occur during aging, and decreases may aggravate age-related diseases. ${ }^{23,24}$ B-ASC and W-ASC transplantation were both followed by increases levels of TSH and SHBG, but the increase associated with B-ASCs was smaller than that following W-ASC 
A

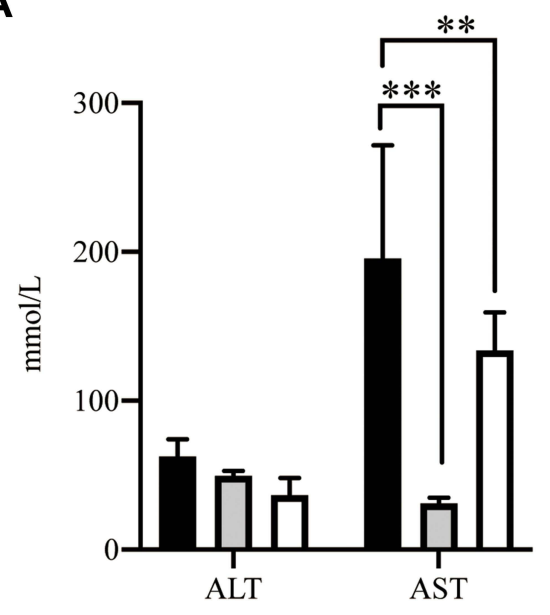

C

$\mathrm{H} \& \mathrm{E}$

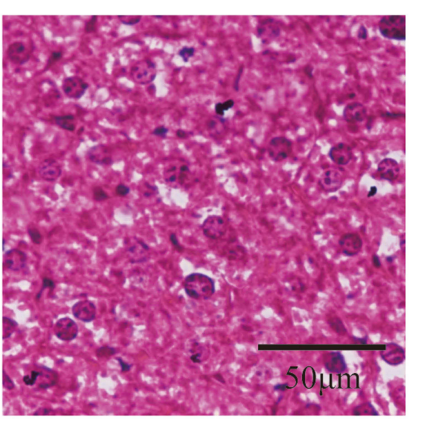

Control

D

Oil Red O

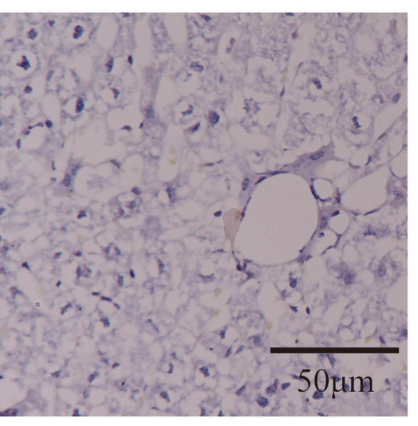

Control

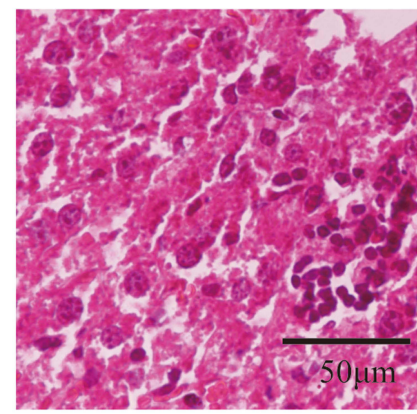

Trans B-ASCs

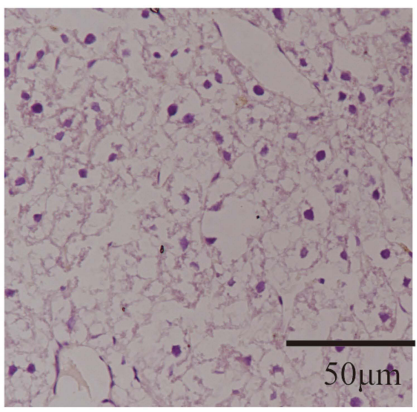

Trans B-ASCs

\section{B}

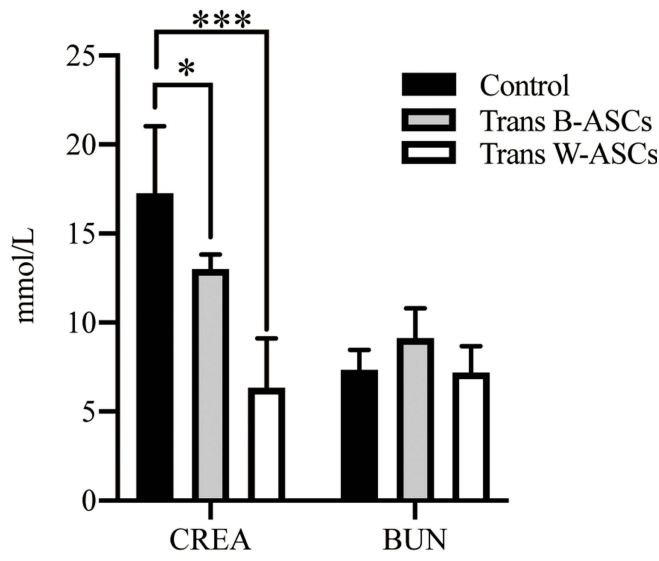

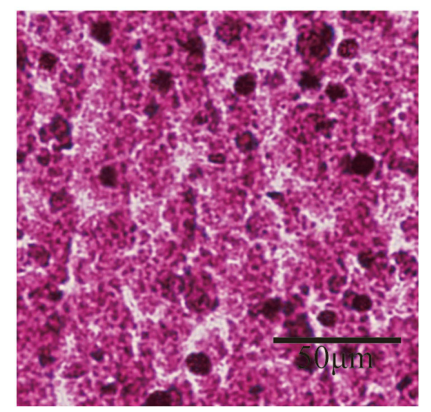

Trans W-ASCs

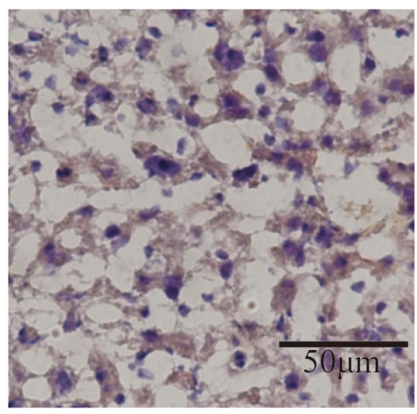

Trans W-ASCs

Figure 6 Effects of B-ASC and W-ASC transplantation on liver and kidney function. (A) ALT and AST levels. (B) CREA and BUN levels. (C) Liver tissue, hematoxylin and eosin staining of paraffin sections. (D) Frozen sections of liver tissue, Oil Red $O$ staining. Data are means \pm standard deviation $(n=5)$. Statistical analysis was conducted with GraphPad Prism 9. $* P<0.05$; $* * P<0.01$; and $* * * P<0.001$.

transplantation. Both B-ASCs and W-ASCs significantly reduced blood glucose levels in aging mice, which may have been related to enhanced mitochondrial activity in adipose tissue and reduced insulin resistance. ${ }^{25}$ LEP levels were significantly increased by W-ASC but not by B-ASCs, suggesting changes in TSH because leptin and TSH are correlated. ${ }^{26}$
Our study found that B-ASCs and W-ASCs have antiaging and antiobesity effects, and that transplantation of B-ASCs and W-ASCs did not cause liver or kidney damage in the aging mice. Liver and kidney function were improved to a certain extent, which is consistent with other studies. ${ }^{27}$ Briefly, ASC isolated from different tissues had heterogeneous antiaging and 
antiobesity effects, but the causes of such differences are not clear. Theoretically, because of brown fat thermogenesis, ASCs derived from brown fat would have better antiobesity effects, but we did not observe that. The superior antiaging and obesity-resistance effects of W-ASCs may have resulted from their anti-inflammatory and antioxidant effects, and their effects on hormone secretion, but data to support that view are lacking.

\section{Conclusion}

This study compared the effects of ASCs derived from white and brown adipose tissue on aging and age-related obesity in a mouse model. The effect of inguinal W-ASCs on body weight, age-related changes in enzymes, hormones, and inflammatory factors was better than that of scapular B-ASCs. Differences in the tissue of origin were reflected by the heterogeneity of the responses to ASCs in aging and agerelated obesity. The study provides a theoretical basis for the future clinical use of ASCs in aging and aging-related obesity.

\section{Acknowledgments}

This study was supported by grants from National Natural Science Foundation of China (32160170 to Qiong Wu), Natural Science Foundation of Guangxi (2020GXNSF AA259049 to QiongWu), Guangxi distinguished expert funding (H.Z.), Innovation Project of Guangxi Graduate Education (YCSW2021115 to Yu Zhu). We also thank International Science Editing for editing this manuscript. Yu Zhu and Tao Wang are co-first authors for this study.

\section{Disclosure}

The authors report no conflicts of interest in this work.

\section{References}

1. Turnheim K. When drug therapy gets old: pharmacokinetics and pharmacodynamics in the elderly. Exp Gerontol. 2003;38 (8):843-853. doi:10.1016/S0531-5565(03)00133-5

2. Tchkonia T, Morbeck DE, Von Zglinicki T, et al. Fat tissue, aging, and cellular senescence. Aging Cell. 2010;9(5):667-684. doi:10.1111/ j.1474-9726.2010.00608.x

3. Akella R, Moon TM, Goldsmith EJ. Unique MAP Kinase binding sites. Biochim Biophys Acta. 2008;1784(1):48-55. doi:10.1016/j. bbapap.2007.09.016

4. Minteer D, Marra KG, Rubin JP. Adipose-derived mesenchymal stem cells: biology and potential applications. In: Weyand B, Dominici M, Hass R, Jacobs R, Kasper C, editors. Mesenchymal Stem Cells - Basics and Clinical Application I. Springer Berlin Heidelberg; 2013:59-71.

5. Bakker GJ, Schnitzler JG, Bekkering S, et al. Oral vancomycin treatment does not alter markers of postprandial inflammation in lean and obese subjects. Physiol Rep. 2019;7(16):e14199. doi:10.14814/ phy2.14199
6. Schoettl T, Fischer IP, Ussar S. Heterogeneity of adipose tissue in development and metabolic function. J Exp Biol. 2018;221(PtSuppl 1). doi: $10.1242 / \mathrm{jeb} .162958$

7. Müller S, Kulenkampff E, Wolfrum C. Adipose Tissue Stem Cells. In: Herzig S, editor. Metabolic Control. Springer International Publishing; 2016:251-263.

8. Chun SY, Lim JO, Lee EH, et al. Preparation and characterization of human adipose tissue-derived extracellular matrix, growth factors, and stem cells: a concise review. Tissue Eng Regen Med. 2019;16 (4):385-393. doi:10.1007/s13770-019-00199-7

9. Zhang D, He S, Wang Q, Pu S, Wu Q. Impact of Aging on the Characterization of Brown and White Adipose Tissue-Derived Stem Cells in Mice. Cells Tissues Organs. 2020;2:1-11.

10. Hye-Youn S, Hahn-Sol B, Youngsook S, Sundong K, Hyun-Sook H, JiUng P. Biological Aging Parameters Can Be Improved After Autologous Adipose-Derived Stem Cell Injection. J Craniofac Surg. 2019;30(3):541.

11. Qiong W, Shuangli H, Yu Z, Shiming P, Zuping Z. Antiobesity Effects of Adipose-Derived Stromal/Stem Cells in a Naturally Aged Mouse Model. Obesity. 2020;29(1):12-54.

12. Jura M, Kozak LP. Obesity and related consequences to ageing. Age. 2016;38(1):23. doi:10.1007/s11357-016-9884-3

13. Tam BT, Morais JA, Santosa S. Obesity and ageing: two sides of the same coin. Obes Rev. 2020;21(4):e12991. doi:10.1111/obr.12991

14. Sendama W. The effect of ageing on the resolution of inflammation. Ageing Res Rev. 2020;57:101000. doi:10.1016/j.arr.2019.101000

15. Buzhor E, Leshansky L, Blumenthal J, et al. Cell-based therapy approaches: the hope for incurable diseases. Regen Med. 2014;9 (5):649-672. doi:10.2217/rme.14.35

16. Campbell A, Brieva T, Raviv L, et al. Concise Review: process Development Considerations for Cell Therapy. Stem Cells Transl Med. 2015;4(10):1155-1163. doi:10.5966/sctm.2014-0294

17. Wang $\mathrm{CH}$, Lundh $\mathrm{M}, \mathrm{Fu} \mathrm{A}$, et al. CRISPR-engineered human brown-like adipocytes prevent diet-induced obesity and ameliorate metabolic syndrome in mice. Sci Transl Med. 2020;12(558). doi:10.1126/scitranslmed.aaz8664

18. Albayrak E, Server S. The relationship of spleen stiffness value measured by shear wave elastography with age, gender, and spleen size in healthy volunteers. J Med Ultrason. 2019;46(2):195-199. doi:10.1007/s10396-019-00929-3

19. Aw D, Hilliard L, Nishikawa Y, Cadman ET, Lawrence RA, Palmer DB. Disorganization of the splenic microanatomy in ageing mice. Immunology. 2016;148(1):92-101. doi:10.1111/imm. 12590

20. Kim IH, Xu J, Liu X, et al. Aging increases the susceptibility of hepatic inflammation, liver fibrosis and aging in response to high-fat diet in mice. Age. 2016;38(4):291-302. doi:10.1007/s11357-016-9938-6

21. Luo J, Mills K, le Cessie S, Noordam R, van Heemst D. Ageing, age-related diseases and oxidative stress: what to do next? Ageing Res Rev. 2020;57:100982. doi:10.1016/j.arr.2019.100982

22. Denu RA, Hematti P. Effects of Oxidative Stress on Mesenchymal Stem Cell Biology. Oxid Med Cell Longev. 2016;2016:2989076. doi:10.1155/2016/2989076

23. Chahal HS, Drake WM. The endocrine system and ageing. $J$ Pathol. 2007;211(2):173-180. doi:10.1002/path.2110

24. Aguiar-Oliveira MH, Bartke A. Growth Hormone Deficiency: health and Longevity. Endocr Rev. 2019;40(2):575-601. doi:10.1210/ er.2018-00216

25. Su T, Xiao Y, Xiao Y, et al. Bone Marrow Mesenchymal Stem Cells-Derived Exosomal MiR-29b-3p Regulates Aging-Associated Insulin Resistance. ACS Nano. 2019;13(2):2450-2462. doi:10.1021/ acsnano.8b09375

26. Mullur R, Liu YY, Brent GA. Thyroid hormone regulation of metabolism. Physiol Rev. 2014;94(2):355-382. doi:10.1152/physrev.00030.2013

27. Börger V, Bremer M, Ferrer-Tur R, et al. Mesenchymal Stem/Stromal Cell-Derived Extracellular Vesicles and Their Potential as Novel Immunomodulatory Therapeutic Agents. Int J Mol Sci. 2017;18 (7):1450. doi:10.3390/ijms18071450 


\section{Publish your work in this journal}

Diabetes, Metabolic Syndrome and Obesity: Targets and Therapy is an international, peer-reviewed open-access journal committed to the rapid publication of the latest laboratory and clinical findings in the fields of diabetes, metabolic syndrome and obesity research. Original research, review, case reports, hypothesis formation, expert opinion and commentaries are all considered for publication. The manuscript management system is completely online and includes a very quick and fair peer-review system, which is all easy to use. Visit http://www.dovepress.com/testimonials.php to read real quotes from published authors. 